\title{
A HOLOGRAPHIC PRINCIPLE FOR THE EXISTENCE OF PARALLEL SPINOR FIELDS AND AN INEQUALITY OF SHI-TAM TYPE*
}

\author{
OUSSAMA HIJAZI ${ }^{\dagger}$ AND SEBASTIÁN MONTIEL MA $^{\ddagger}$
}

\begin{abstract}
Suppose that $\Sigma=\partial M$ is the $n$-dimensional boundary of a connected compact Riemannian spin manifold $(M,\langle\rangle$,$) with non-negative scalar curvature, and that the (inward) mean$ curvature $H$ of $\Sigma$ is positive. We show that the first eigenvalue of the Dirac operator of the boundary corresponding to the conformal metric $\langle,\rangle_{H}=H^{2}\langle$,$\rangle is at least n / 2$ and equality holds if and only if there exists a non-trivial parallel spinor field on $M$. As a consequence, if $\Sigma$ admits an isometric and isospin immersion $F$ with mean curvature $H_{0}$ as a hypersurface into another spin Riemannian manifold $M_{0}$ admitting a parallel spinor field, then
\end{abstract}

$$
\int_{\Sigma} H d \Sigma \leq \int_{\Sigma} \frac{H_{0}^{2}}{H} d \Sigma
$$

and equality holds if and only if both immersions have the same shape operator. In this case, $\Sigma$ has to be also connected. In the special case where $M_{0}=\mathbb{R}^{n+1}$, equality in (1) implies that $M$ is a Euclidean domain and $F$ is congruent to the embedding of $\Sigma$ in $M$ as its boundary. We also prove that Inequality (1) implies the Positive Mass Theorem (PMT).

Key words. Manifolds with boundary, Dirac operator, eigenvalues, rigidity, Positive Mass Theorem.

AMS subject classifications. 53C27, 53C40, 53C80, 58G25.

1. Introduction. In [ST1], Shi and Tam used the PMT to study the boundary behavior of compact Riemannian spin manifolds with non-negative scalar curvature. More precisely, they proved the following:

Let $M$ be an $(n+1)$-dimensional compact connected Riemannian spin manifold with non-negative scalar curvature and mean convex boundary hypersurface $\Sigma$. If $\Sigma$ admits an isometric strictly convex immersion $F$ into $\mathbb{R}^{n+1}$, then

$$
\int_{\Sigma} H d \Sigma \leq \int_{\Sigma} H_{0} d \Sigma
$$

where $H$ is the mean curvature of $\Sigma$ as the boundary of $M$ and $H_{0}$ stands for the mean curvature of the immersion $F$ of $\Sigma$ into $\mathbb{R}^{n+1}$. Equality holds if and only if $\Sigma$ is connected, $M$ is a Euclidean domain and the embedding of $\Sigma$ in $M$ and its immersion in $\mathbb{R}^{n+1}$ are congruent.

For $n=2$, by the Weyl Embedding Theorem, the assumption that the boundary $\Sigma$ embeds isometrically as a strictly convex hypersurface in $\mathbb{R}^{3}$ is equivalent to the fact that $\Sigma$ has positive Gauss curvature. Hence, Inequality (2) implies that positively curved mean convex boundaries in time-symmetric initial data sets, satisfying the dominant energy condition, have non-negative Brown-York Mass (see $[\mathrm{BY}])$. Note that, a generalization of this result, to subsets of general data sets, is

\footnotetext{
*Received December 21, 2012; accepted for publication March 8, 2013.

†Institut Élie Cartan de Lorraine, Université de Lorraine, Nancy, B.P. 239, 54506 VandœuvreLès-Nancy Cedex, France (Oussama.Hijazi@univ-lorraine.fr).

¥Departamento de Geometría y Topología, Universidad de Granada, 18071 Granada, Spain (smon tiel@ugr.es). The second author was partially supported by a Spanish MEC-FEDER grant No. MTM2007-61775.
} 
given in $[\mathrm{LY} 1, \mathrm{LY} 2]$ and in $[\mathrm{WY}]$ for the hyperbolic setting.

In this paper, we prove the following main results:

Theorem 1. Let $(M,\langle\rangle$,$) be an (n+1)$-dimensional compact connected Riemannian spin manifold with non-negative scalar curvature and with mean convex boundary hypersurface $\Sigma$. Then, the first non-negative eigenvalue $\lambda_{1}\left(\not D_{H}\right)$ of the Dirac operator corresponding to the conformal metric $\langle,\rangle_{H}=H^{2}\langle$,$\rangle satisfies$

$$
\lambda_{1}\left(\not D_{H}\right) \geq \frac{n}{2}
$$

and equality holds if and only if $M$ admits a non trivial parallel spinor. In this case, the eigenspace corresponding to $\lambda_{1}\left(\not_{H}\right)=\frac{n}{2}$ consists of restrictions to $\Sigma$ of parallel spinor fields on $M$ multiplied by the function $H^{-\frac{n-1}{2}}$. Furthermore, the boundary hypersurface $\Sigma$ has to be connected.

Making use of the restriction to the hypersurface of a parallel spinor field, we get:

THEOREM 2. Under the same conditions as Theorem 1, assume furthermore that $\Sigma$ admits an isometric and isospin immersion into another $(n+1)$-dimensional Riemannian spin manifold $M_{0}$ endowed with a non trivial parallel spinor field.Then Inequality (1) holds. Moreover, equality is achieved if and only if both immersions have the same shape operator. In this case, the boundary hypersurface $\Sigma$ must be connected.

If we choose $M_{0}=\mathbb{R}^{n+1}$ in Theorem 2 , then the equality case could be considerably improved. We have:

THEOREM 3. Under the same conditions as Theorem 2, assume that $M_{0}=\mathbb{R}^{n+1}$. Then Inequality (1) holds and equality is achieved if and only if $\Sigma$ is connected, $M$ is a Euclidean domain and the embedding of $\Sigma$ in $M$ and its immersion in $\mathbb{R}^{n+1}$ are congruent.

As we mentioned, Inequality (2) is valid [ST1, Theorem 4.1] under the hypothesis that the isometric immersion $F$ is the inclusion map of a strictly convex hypersurface $\Sigma \subset \mathbb{R}^{n+1}$ (note that, due to its convexity, each connected component of $\Sigma$ must be diffeomorphic to an $n$-dimensional sphere and so $\Sigma$ admits a unique spin structure). Now, combine a Schwarz inequality and Inequality (2), to get

$$
\left(\int_{\Sigma} H_{0} d \Sigma\right)^{2} \leq \int_{\Sigma} \frac{H_{0}^{2}}{H} d \Sigma \int_{\Sigma} H d \Sigma \leq \int_{\Sigma} \frac{H_{0}^{2}}{H} d \Sigma \int_{\Sigma} H_{0} d \Sigma .
$$

Since $F$ is a strictly convex immersion, we have that the mean curvature $H_{0}$ of $F$ is positive. Thus, from Inequality (4), we deduce

$$
\int_{\Sigma} H_{0} d \Sigma \leq \int_{\Sigma} \frac{H_{0}^{2}}{H} d \Sigma .
$$

(Observe that we can also deduce Inequality (5) by combining the first inequality in (4) and Inequality (1), provided that the integral of $H_{0}$ on $\Sigma$ is positive). Inequality (5) together with (2) imply Inequality (1). However, Theorem 3 is valid in a more general setup than that of Inequality (2) (and its generalizations, such that obtained in $[\mathrm{EMW}])$, since no convexity assumptions on the immersion $F$ is imposed. 
Another important remark about the relationship between Theorem 3 and [ST1, Theorem 4.1] is that a key ingredient in their proof is a version of the PMT (see [SY]) for $C^{2}$-metrics. Furthermore, Shi and Tam showed (see [ST1, Theorem 5.1]) that Inequality (2) also implies the PMT (at least in the 3-dimensional case, the more significant from the physical point of view). Recall that the proof of Inequality (1) makes no use of the PMT. However, even though it is weaker than Inequality (2), it implies the PMT (see Corollary 10).

2. Preliminaries on spin manifolds. Let $(M,\langle\rangle$,$) be an (n+1)$-dimensional Riemannian spin manifold, which we will suppose from now on to be connected, and denote by $\nabla$ the Levi-Civita connection on its tangent bundle $T M$. We choose a spin structure on $M$ and consider the corresponding spinor bundle $\mathbb{S} M$ which is a rank $2^{\left[\frac{n+1}{2}\right]}$ complex vector bundle. Denote by $\gamma$ the Clifford multiplication

$$
\gamma: \mathbb{C} \ell(M) \longrightarrow \operatorname{End}(\mathbb{S} M)
$$

which is a fibre preserving algebra morphism. Then $\mathbb{S} M$ becomes a bundle of complex left modules over the Clifford bundle $\mathbb{C} \ell(M)$ over the manifold $M$. When $(n+1)$ is even, the spinor bundle splits into the direct sum of the positive and negative chiral subbundles

$$
\mathbb{S} M=\mathbb{S} M^{+} \oplus \mathbb{S} M^{-}
$$

where $\mathbb{S} M^{ \pm}$are defined to be the \pm 1 -eigenspaces of the endomorphism $\gamma\left(\omega_{n+1}\right)$, with $\omega_{n+1}=i^{\left[\frac{n+2}{2}\right]} e_{1} \cdot e_{2} \cdots e_{n+1}$ the complex volume form.

On the spinor bundle $\mathbb{S} M$, one has (see [LM]) a natural Hermitian metric, denoted as the Riemannian metric on $M$ by $\langle$,$\rangle , and the spinorial Levi-Civita connection \nabla$ acting on spinor fields. The Hermitian metric and $\nabla$ are compatible with the Clifford multiplication (6) and compatible with each other. That is

$$
\begin{gathered}
\langle\gamma(X) \psi, \gamma(X) \varphi\rangle=|X|^{2}\langle\psi, \varphi\rangle \\
X\langle\psi, \varphi\rangle=\left\langle\nabla_{X} \psi, \varphi\right\rangle+\left\langle\psi, \nabla_{X} \varphi\right\rangle \\
\nabla_{X}(\gamma(Y) \psi)=\gamma\left(\nabla_{X} Y\right) \psi+\gamma(Y) \nabla_{X} \psi
\end{gathered}
$$

for any spinor fields $\psi, \varphi \in \Gamma(\mathbb{S} M)$ and any tangent vector fields $X, Y \in \Gamma(T M)$. Since $\nabla \omega_{n+1}=0$, for $(n+1)$ even, the decomposition (7) becomes orthogonal and $\nabla$ preserves this decomposition.

The Dirac operator $D$ on $\mathbb{S} M$ is the first order elliptic differential operator locally given by

$$
D=\sum_{i=1}^{n+1} \gamma\left(e_{i}\right) \nabla_{e_{i}}
$$

where $\left\{e_{1}, \ldots, e_{n+1}\right\}$ is a local orthonormal frame of $T M$. When $(n+1)$ is even, the Dirac operator interchanges positive and negative spinor fields, that is,

$$
D: \Gamma\left(\mathbb{S} M^{ \pm}\right) \longmapsto \Gamma\left(\mathbb{S} M^{\mp}\right) .
$$

3. Hypersurfaces and induced structures. In this section, we compare the

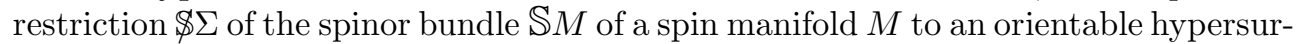
face $\Sigma$ immersed into $M$ and its Dirac-type operator $\not D$ to the intrinsic spinor bundle 
$\mathbb{S} \Sigma$ of the induced spin structure on $\Sigma$ and its fundamental Dirac operator $D_{\Sigma}$. A fundamental case will be when the hypersurface $\Sigma$ is just the boundary $\partial M$ of a manifold $M$ with non empty boundary. These facts are in general well-known (see for example [Bu, Tr, Bä2, BFGK] or our previous papers [HMZ1, HMZ2, HMZ3, HMR, HM]). For completeness, we introduce the notations and the key facts.

Denote by $\nabla$ the Levi-Civita connection associated with the induced Riemannian metric on $\Sigma$. The Gauß formula says that

$$
\nabla_{X} Y=\nabla_{X} Y-\langle A X, Y\rangle N
$$

where $X, Y$ are vector fields tangent to the hypersurface $\Sigma$, the vector field $N$ is a global unit field normal to $\Sigma$ and $A$ stands for the shape operator corresponding to $N$, that is,

$$
\nabla_{X} N=-A X, \quad \forall X \in \Gamma(T \Sigma) .
$$

We have that the restriction

$$
\$ \Sigma:=\mathbb{S} M_{\mid \Sigma}
$$

is a left module over $\mathbb{C} \ell(\Sigma)$ for the induced Clifford multiplication

$$
\not /: \mathbb{C} \ell(\Sigma) \longrightarrow \operatorname{End}(\$ \Sigma)
$$

given by

$$
\not(X) \psi=\gamma(X) \gamma(N) \psi
$$

for every $\psi \in \Gamma(\$ \$ \$)$ and $X \in \Gamma(T \Sigma)$ (note that a spinor field on the ambient manifold $M$ and its restriction to the hypersurface $\Sigma$ will be denoted by the same symbol). Consider on $\$ \Phi$ the Hermitian metric $\langle$,$\rangle induced from that of \mathbb{S} M$. This metric immediately satisfies the compatibility condition (8) if one considers on $\Sigma$ the Riemannian metric induced from $M$ and the Clifford multiplication $\not /$ defined in (15). Now the Gauss formula (12) implies that the spin connection $\not \nabla$ on $\$ \$$ is given by the following spinorial Gauss formula

$$
\nabla_{X} \psi=\nabla_{X} \psi-\frac{1}{2} \not(A X) \psi=\nabla_{X} \psi-\frac{1}{2} \gamma(A X) \gamma(N) \psi
$$

for every $\psi \in \Gamma(\$ \Phi \Sigma)$ and $X \in \Gamma(T \Sigma)$. Note that the compability conditions (8), (9) and $(10)$ are satisfied by $(\$ \$ \Sigma, \not,\langle\rangle,, \not{\nabla})$.

Denote by $\not D: \Gamma(\$ \$ \$) \rightarrow \Gamma(\$ \Sigma)$ the Dirac operator associated with the Dirac bundle $\$ \Sigma$ over the hypersurface. It is a well-known fact that $\not D$ is a first order elliptic differential operator which is formally $L^{2}$-selfadjoint. By (16), for any spinor field $\psi \in \Gamma(\mathbb{S} M)$, we have

$$
\not D \psi=\sum_{j=1}^{n} \not\left(e_{j}\right) \nabla_{e_{j}} \psi=\frac{n}{2} H \psi-\gamma(N) \sum_{j=1}^{n} \gamma\left(e_{j}\right) \nabla_{e_{j}} \psi,
$$

where $\left\{e_{1}, \ldots, e_{n}\right\}$ is a local orthonormal frame of $T \Sigma$ and $H=\frac{1}{n} \operatorname{trace} A$ is the mean curvature of $\Sigma$ corresponding to the orientation $N$. Using (16) and (13), it is straightforward to see that the skew-commutativity rule

$$
\not D(\gamma(N) \psi)=-\gamma(N) \not D \psi
$$


holds for any spinor field $\psi \in \Gamma(\mathbb{\$} \Sigma)$. It is important to point out that, from this fact, the spectrum of $\not D$ is always symmetric with respect to zero, while this is the case for the Dirac operator $D_{\Sigma}$ of the intrinsic spinor bundle only when $n$ is even. Indeed, in this case, we have an isomorphism of Dirac bundles

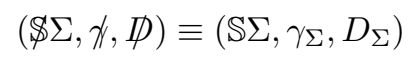

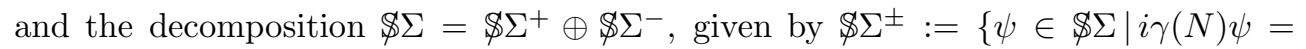
$\pm \psi\}$, corresponds to the chiral decomposition of the spinor bundle $\mathbb{S} \Sigma$. Hence $\not D$

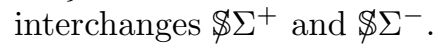

When $n$ is odd the spectrum of $D_{\Sigma}$ is not necessarily symmetric. In fact, in this case, the spectrum of $\not D$ is just the symmetrization of the spectrum of $D_{\Sigma}$. This is why the decomposition of $\mathbb{S} M$ into positive and negative chiral spinors induces

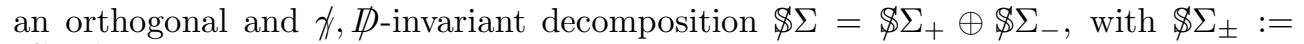
$\left(\mathbb{S} M^{ \pm}\right)_{\mid \Sigma}$, in such a way that

$$
\left(\mathbb{S} \Sigma_{ \pm}, \not /, \not \phi_{\mid \not \Sigma_{ \pm}}\right) \equiv\left(\mathbb{S} \Sigma, \pm \gamma_{\Sigma}, \pm D_{\Sigma}\right) .
$$

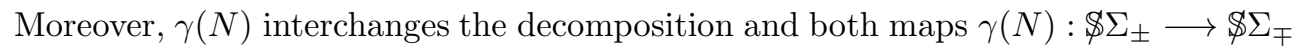
are isomorphisms.

Consequently, to study the spectrum of the induced operator $\not D$ is equivalent to study the spectrum of the Dirac operator $D_{\Sigma}$ of the spin Riemannian structure induced on the hypersurface $\Sigma$.

4. Conformal covariance of the Dirac operator. Consider a positive function $h$ on a Riemannian spin $n$-dimensional manifold $\Sigma$ and the corresponding conformal metric $\langle,\rangle^{\star}=h^{2}\langle$,$\rangle . We know that there exists (see [Hit, Hij, BHMMM])$

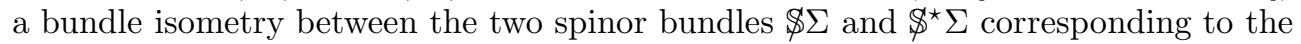
same spin structure and to the two conformally related metrics. For this reason, the two spinor bundles will be denoted by the same symbol $\$ \Sigma$. With this identification in mind, for the corresponding Clifford multiplications and spin connections, one has:

$$
\not^{\star}=h \psi, \quad \nabla_{X}^{\star}-\nabla_{X}=-\frac{1}{2 h} \not(X) \not(\nabla h)-\frac{1}{2 h}\langle X, \nabla h\rangle,
$$

for all $X \in \Gamma(T \Sigma)$. We can easily find from (19) the relation between the two Dirac operators $\not D$ and $\not D^{\star}$ on $\$ \Sigma$ relative to the two conformally related metrics on $M$. For any spinor field $\psi \in \Gamma(\$ \Phi \Sigma)$, one has:

$$
\not D^{\star}\left(h^{-\frac{n-1}{2}} \psi\right)=h^{-\frac{n+1}{2}} \not D \psi .
$$

5. A Reilly inequality for manifolds with boundary. Another key fact we will need is the following spinorial Reilly type inequality, valid when the manifold $M$ is compact. By integration of the well-known Schrödinger-Lichnerowicz formula over the compact $(n+1)$-dimensional Riemannian spin manifold $M$ with boundary $\Sigma=\partial M$ and using a standard Schwarz inequality involving the lengths of the spin Levi-Civita connection and of the Dirac operator of $M$ (see for instance [HMZ1]), for any spinor field $\psi \in \Gamma(\mathbb{S} M)$, one has

$$
\begin{gathered}
\int_{\Sigma}\left(\langle\not D \psi, \psi\rangle-\frac{n H}{2}|\psi|^{2}\right) d \Sigma \geq \\
\frac{1}{4} \int_{M} R|\psi|^{2} d M-\frac{n}{n+1} \int_{M}|D \psi|^{2} d M,
\end{gathered}
$$

where $R$ is the scalar curvature on $M$. It is a well-known fact that equality occurs if and only if $\psi$ is a twistor-spinor (see [BFGK] for the corresponding definition) on $M$. 
6. A local boundary elliptic condition for the Dirac operator. As before, $\Sigma$ is the boundary hypersurface of an $(n+1)$-dimensional Riemannian spin compact manifold $M$. We define two pointwise projections

$$
P_{ \pm}: \$ \Phi \Sigma \longrightarrow \$
$$

on the induced Dirac bundle over the hypersurface, as follows

$$
P_{ \pm}=\frac{1}{2}\left(\operatorname{Id}_{\$ \Sigma} \pm i \gamma(N)\right)
$$

Note that, as pointed out at the end of Section 3, when $n$ is even, these are nothing

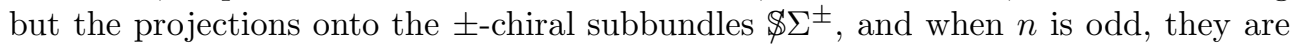
the projections onto the restrictions to $\Sigma$ of the chiral subbundles $\mathbb{S} M^{ \pm}$of the evendimensional manifold $M$. It is immediate to see that $P_{+}$and $P_{-}$are selfadjoint and

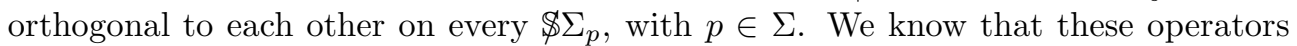
provide good (local) boundary conditions to solve equations for the Dirac operator $D$ of $M$. In fact, it is well-known (at least when the metric of $M$ is cylindrical near $\Sigma$ ) that the boundary conditions $P_{ \pm}=0$, which are sometimes called MIT bag conditions ([CJJT, CJJTW, J]), satisfy the Lopatinsky-Shapiro condition for ellipticity (see [Hö] and [BW, Chapter 18] for a definition, and [HMR, HMZ3] for details and applications). Although the ellipticity of these boundary conditions $P_{ \pm}=0$ is proved only in the cylindrical case and extensively used in the general case, we refer to [BäBa, Section 7, particularly Example 7.26] in order to check that this general use is correct (see also $[\mathrm{BC}])$. These facts can be summarized as follows.

Proposition 4. Let $M$ be a compact Riemannian spin manifold with boundary $\partial M=\Sigma$. Then the two orthogonal projections $P_{ \pm}$acting on the spin bundle $\$ \Sigma$, defined in (22), induced on $\Sigma$ from the spin bundle $\mathbb{S} M$ of a given spin structure on $M$, provide (local) elliptic boundary conditions for the Dirac operator $D$ of $M$.

REMARK 1. Even though in the literature one can frequently find the assertion that the classical Dirac operator D does not admit elliptic boundary conditions in any dimension and that, in fact, there are topological obstructions for its existence when the dimension of $\Sigma$ is odd [BW, GLP, HMZ2, Se], we have to point out that our local boundary conditions provided by the projections $P_{ \pm}$exist for all dimensions without any restriction. This is due to the fact that, when $n$ is odd, the induced spin bundle

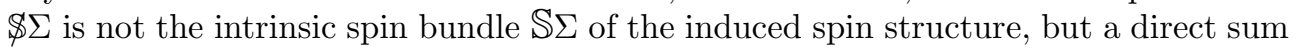
of two copies of it, since we are considering the total spin bundle $\mathbb{S} M$ on the ambient manifold instead of one of its chiral subbundles. Then, in this case, the Dirac operator $D$ is really a pair of classical chiral Dirac operators and the usual restrictions do not apply to this situation. Note also that the Green integral formula

$$
\int_{M}\langle D \psi, \varphi\rangle d M-\int_{M}\langle\psi, D \varphi\rangle d M=\int_{\Sigma}\langle\psi, \gamma(N) \varphi\rangle d \Sigma,
$$

where $\psi, \varphi \in \Gamma(\mathbb{S} M)$, shows that none of the conditions provided by $P_{ \pm}$makes $D$ a formally selfadjoint operator. Instead, one can easily see that the boundary realizations $\left(D, P_{+}\right)$and $\left(D, P_{-}\right)$of $D$ are adjoint to each other.

The ellipticity of the boundary conditions given by $P_{+}$and $P_{-}$and that of the Dirac operator $D$ of $M$ allow to solve boundary value problems for $D$ on $M$ prescribing on the boundary $\Sigma$ the corresponding $P_{ \pm}$-projections of the solutions. For completeness, we give a proof. 
Proposition 5. The following two types of inhomogeneous problems for the Dirac operator $D$ of a compact Riemannian spin manifold $M$, with boundary a hypersurface $\Sigma$,

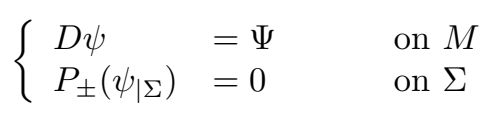

have a unique smooth solution for any $\Psi \in \Gamma(\mathbb{S} M)$.

Proof. The two realizations of $D$ associated with the two boundary conditions $P_{ \pm}$are the two unbounded operators

$$
D_{ \pm}: \operatorname{Dom} D_{ \pm}=\left\{\psi \in H^{1}(\mathbb{S} M) \mid P_{ \pm}\left(\psi_{\mid \Sigma}\right)=0\right\} \longrightarrow L^{2}(\mathbb{S} M)
$$

where $H^{1}(\mathbb{S} M)$ stands for the Sobolev space of $L^{2}$-spinors with weak $L^{2}$ covariant derivatives (recall that such spinors have a well defined $L^{2}$ trace on $\Sigma$ ). From (23) and the end of Remark 1, it follows that for the adjoint, one has $\left(D_{ \pm}\right)^{*}=D_{\mp}$. Moreover, if $\psi \in \operatorname{Dom} D_{ \pm}$is a solution of the corresponding homogeneous problem, that is, if it belongs to $\operatorname{ker} D_{ \pm}$, the ellipticy of both the Dirac operator $D$ and the boundary condition $P_{ \pm}=0$ imply regularity results from which (see [BäBa, Corollary 7.18]) one gets that $\psi$ is smooth. On the other hand, taking $\varphi=i \psi$ in (23) and recalling that the metric on $\mathbb{S} M$ is Hermitian, we have

$$
0=2 \int_{M}\langle D \psi, i \psi\rangle d M=\int_{\Sigma}\langle\psi, i \gamma(N) \psi\rangle d \Sigma=\mp \int_{\Sigma}|\psi|^{2} d \Sigma .
$$

Then one sees that the smooth harmonic spinor $\psi$ on the compact manifold $M$ has a vanishing trace $\psi_{\mid \Sigma}$ along the boundary hypersurface $\Sigma$. But according to [Bä1], in an $(n+1)$-dimensional manifold like $M$, the Haussdorf measure of the zero set of a smooth non trivial harmonic spinor must be less than or equal to $n-1$. So, the spinor field $\psi$ vanishes on the whole of $M$. Then

$$
\operatorname{ker} D_{ \pm}=\{0\} \quad \text { and } \quad \operatorname{coker} D_{ \pm} \cong \operatorname{ker}\left(D_{ \pm}\right)^{*}=\operatorname{ker} D_{\mp}=\{0\} .
$$

Then the two realizations $D_{ \pm}$are invertible operators, hence if $\Psi \in \Gamma(\mathbb{S} M)$ is a smooth spinor field on $M$, there exists a unique solution $\psi \in H^{1}(\mathbb{S} M)$ of $(24)$. Now, Proposition 4 and the regularity results proved in [BäBa, Theorem 7.17] imply (cf. also [BW, Chapter 19]) the required smoothness of the solution $\psi$.

REMARK 2. When the dimension $(n+1)$ of the manifold $M$ is odd, the final considerations made in Section 3 along with Proposition 5 give the existence, uniqueness and the regularity to the two problems

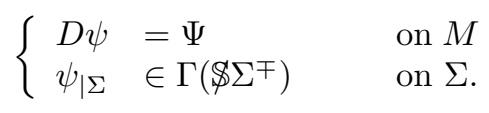

In contrast, when $(n+1)$ is even, we may decompose the given spinor fields $\psi$ and $\Psi$ according to the chiral subbundles (7) of $M$. Thus Proposition 5 solves the following boundary first order system

$$
\left\{\begin{array}{lll}
D \psi_{ \pm} & =\Psi_{\mp} & \text { on } M \\
i \gamma(N)\left(\psi_{ \pm \mid \Sigma}\right) & =\mp \psi_{ \pm \mid \Sigma} & \text { on } \Sigma
\end{array}\right.
$$

where now all the involved fields have a fixed chirality. 
Proposition 6. [HMZ3] Let $M$ be a compact Riemannian spin manifold with boundary, a hypersurface $\Sigma$. If $\varphi \in \Gamma(\$ \$ \$)$ is a smooth spinor field of the induced Dirac bundle and $\Psi \in \Gamma(\mathbb{S} M)$, then the following boundary problem for the Dirac operator

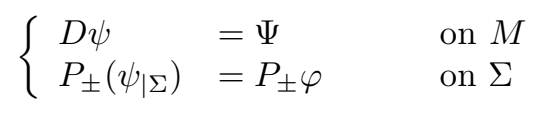

has a unique smooth solution $\psi \in \Gamma(\mathbb{S} M)$.

Proof. Extend $\varphi$ to a spinor field $\widehat{\varphi} \in \Gamma(\mathbb{S} M)$ and put $\widehat{\psi}=\psi-\widehat{\varphi}$. Then solve

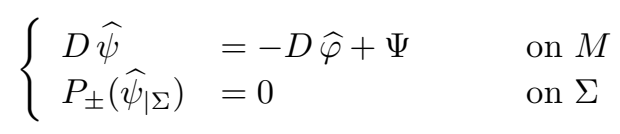

using Proposition 5.

REMARK 3. Note that when one considers the well-known elliptic global APS boundary condition, introduced by Atiyah, Patodi and Singer in order to study a version of the Index Theorem in the case of manifolds with non empty boundary (see [APS]), the boundary problems for the Dirac operator corresponding to those solved in Propositions 5 and 6 do not necessarily have solutions. From the spinorial proofs of the Positive Mass Theorem (see for instance [He, Wi]), it appears that for solving boundary problems with the APS boundary condition, it is necessary to impose some non negativity condition on the scalar curvature of $M$ and some lower estimate on the mean curvature of the boundary hypersurface (see also [HMR] for other types of boundary conditions).

7. A holographic principle for the existence of parallel spinors. It is by now a known approach (see [HMZ2, HMZ3]) to make use of the Reilly type inequality (21) for a compact Riemannian spin manifold $M$ with non-negative scalar curvature $R$, together with the solution of an appropriate boundary problem for the Dirac operator $D$ of $M$, in order to establish a certain integral inequality $((27)$ in this case) for the induced Dirac operator $\not D$ of the boundary hypersurface $\partial M=\Sigma$. Moreover, we will also assume that the inward mean curvature $H$ of $\Sigma$ is positive, that is, $\Sigma$ is mean convex, and so Inequality (27) will be translated into some results about the first eigenvalue of the Dirac operator $\not_{H}$, associated with the conformal metric $\langle,\rangle_{H}=H^{2}\langle$,$\rangle on \Sigma$. First, we need to recall the following fact:

LEMma 7. For any smooth spinor field $\psi \in \Gamma(\$ \$ \Sigma)$ we have

$$
\int_{\Sigma}\langle\not D \psi, \psi\rangle d \Sigma=2 \int_{\Sigma}\left\langle\not D P_{+} \psi, P_{-} \psi\right\rangle d \Sigma .
$$

Proof. We have the pointwise orthogonal decomposition $\psi=P_{+} \psi+P_{-} \psi$. Moreover, from (18) and (22), one immediately shows that

$$
\not D P_{ \pm}=P_{\mp} \not D
$$

Hence, since $P_{+}$and $P_{-}$are orthogonal to each other,

$$
\langle\not D \psi, \psi\rangle=\left\langle\not D P_{+} \psi, P_{-} \psi\right\rangle+\left\langle\not D P_{-} \psi, P_{+} \psi\right\rangle
$$


We conclude by noting that $\Sigma$ is compact and the operator $\not D$ is formally $L^{2}$ selfadjoint.

Proposition 8. Let $M$ be a compact spin Riemannian manifold with nonnegative scalar curvature, whose boundary hypersurface $\Sigma=\partial M$ has positive (inward) mean curvature $H$. Let $\varphi \in \Gamma(\$ \Sigma)$ be any spinor field on the restricted Dirac bundle. Then

$$
0 \leq \int_{\Sigma}\left(\frac{1}{H}\left|\not D P_{+} \varphi\right|^{2}-\frac{n^{2}}{4} H\left|P_{+} \varphi\right|^{2}\right) d \Sigma .
$$

Moreover, equality holds if and only if there exists a parallel spinor field $\psi \in \Gamma(\mathbb{S} M)$ such that $P_{+} \psi=P_{+} \varphi$ along the boundary hypersurface $\Sigma$.

Proof. As mentioned before, unless otherwise stated, we shall use the same symbols to indicate spinor fields defined on $M$ and their restrictions to the boundary $\Sigma$. Take any spinor field $\varphi \in \Gamma(\$ \Sigma)$ of the induced spin bundle on the hypersurface and consider the following boundary problem

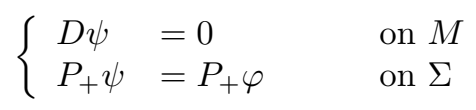

for the Dirac operator $D$ and the boundary condition $P_{+}$. Proposition 6 asserts that this problem has a unique smooth solution $\psi \in \Gamma(\mathbb{S} M)$. Applying the Reilly Inequality (21) to such a spinor field $\psi$ and taking into account that $R \geq 0$ on $M$, we obtain the following key inequality

$$
0 \leq \int_{\Sigma}\left(\langle\not D \psi, \psi\rangle-\frac{n}{2} H|\psi|^{2}\right) d \Sigma
$$

where, if equality is achieved, then $\psi$ is a twistor-spinor field. But it is also harmonic, hence it is parallel. Using Inequality (27) combined with Lemma 7 and the fact that the decomposition

$$
\psi=P_{+} \psi+P_{-} \psi
$$

is pointwise orthogonal, we get

$$
0 \leq \int_{\Sigma}\left(2\left\langle\not D P_{+} \psi, P_{-} \psi\right\rangle-\frac{n}{2} H\left|P_{+} \psi\right|^{2}-\frac{n}{2} H\left|P_{-} \psi\right|^{2}\right) d \Sigma .
$$

The mean curvature $H$ being assumed positive, we consider the obvious pointwise inequality

$$
\begin{gathered}
0 \leq\left|\frac{1}{\sqrt{\frac{n}{2} H}} \not D P_{+} \psi-\sqrt{\frac{n}{2} H} P_{-} \psi\right|^{2}= \\
\frac{1}{\frac{n}{2} H}\left|\not D P_{+} \psi\right|^{2}+\frac{n}{2} H\left|P_{-} \psi\right|^{2}-2\left\langle\not D P_{+} \psi, P_{-} \psi\right\rangle .
\end{gathered}
$$

In other words, we have

$$
2\left\langle\not D P_{+} \psi, P_{-} \psi\right\rangle-\frac{n}{2} H\left|P_{-} \psi\right|^{2} \leq \frac{1}{\frac{n}{2} H}\left|\not D P_{+} \psi\right|^{2},
$$


which, when combined with Inequality (28), implies Inequality (26). Now, in order to study the equality case, recall that the harmonic spinor $\psi$ on $M$ is such that $P_{+} \psi=P_{+} \varphi$. If equality holds, we already know from (27) that the spinor field $\psi$ must be parallel.

Conversely, assume that there is a parallel spinor field $\psi$ on $M$. Then from (17), along the boundary $\Sigma$, we have

$$
\not D \psi=\frac{n}{2} H \psi
$$

Using the relations (25), the previous equality splits into

$$
\not D P_{+} \psi=\frac{n}{2} H P_{-} \psi \quad \text { and } \quad \not D P_{-} \psi=\frac{n}{2} H P_{+} \psi
$$

From these two relations and the formal $L^{2}$ selfadjointness of $\not D$, it is straightforward to see that equality holds in (26) for $P_{+} \psi \in \Gamma(\mathbb{\$} \Sigma)$.

With this, we are ready to state the following key result:

Proposition 9. Let $M$ be a compact spin Riemannian $(n+1)$-dimensional manifold with non-negative scalar curvature, whose boundary hypersurface $\Sigma$ has positive (inward) mean curvature $H$ (that is, $\Sigma$ is mean convex). Then

$$
0 \leq \int_{\Sigma}\left(\frac{1}{H}|\not D \varphi|^{2}-\frac{n^{2}}{4} H|\varphi|^{2}\right) d \Sigma,
$$

for any spinor field $\varphi \in \Gamma(\$ \Sigma)$. Equality holds if and only if there exist two parallel spinor fields $\psi, \Psi \in \Gamma(\mathbb{S} M)$ such that $P_{+} \psi=P_{+} \varphi$ and $P_{-} \Psi=P_{-} \varphi$ on the boundary.

Proof. Since there is an obvious symmetry between the two boundary conditions $P_{+}$and $P_{-}$for the Dirac operator on $M$ (see Proposition 6), one can repeat the proof of Proposition 8 to get the inequality corresponding to (26) where the positive projection $P_{+}$is replaced by the negative one $P_{-}$. Hence, for any spinor field $\varphi \in \Gamma(\$ \Phi)$, we also have

$$
0 \leq \int_{\Sigma}\left(\frac{1}{H}\left|\not D P_{-} \varphi\right|^{2}-\frac{n^{2}}{4} H\left|P_{-} \varphi\right|^{2}\right) d \Sigma .
$$

Taking into account the relation (25) and the pointwise orthogonality of the projections $P_{ \pm}$, the sum of the two inequalities (26) and (30) yields (29). The equality case is a consequence of Proposition 8 .

REMARK 4. Note that, in the case of equality, we cannot conclude that the two parallel spinors in Proposition 9 coincide. In fact, assume that the spin manifold $M$ admits a space of parallel spinor fields with dimension at least 2. Take two different parallel spinor fields $\psi, \Psi \in \Gamma(\mathbb{S} M)$ and define $\varphi=P_{+}\left(\psi_{\mid \Sigma}\right)+P_{-}\left(\Psi_{\mid \Sigma}\right)$. Such a spinor field on the boundary $\Sigma$ achieves the equality in Inequality (29).

Proof of Theorem 1. We consider on the boundary hypersurface $\Sigma$ the conformally modified Riemannian metric $\langle,\rangle_{H}=H^{2}\langle$,$\rangle . Using the conformal covariance of the$ Dirac operator (see (20)) we have that, for any spinor field $\varphi \in \Gamma(\mathbb{\$} \Sigma)$,

$$
\not D_{H} \varphi_{H}=H^{-\frac{n+1}{2}} \not D \varphi
$$


where $\varphi_{H}=H^{-\frac{n-1}{2}} \varphi$ and $\not D_{H}$ is the induced Dirac operator associated with the metric $\langle,\rangle_{H}$. Then, as the Riemannian measures of the two conformally related metrics satisfy

$$
d \Sigma_{H}=H^{n} d \Sigma
$$

we obtain the equalities

$$
\left|\not D_{H} \varphi_{H}\right|^{2} d \Sigma_{H}=\frac{1}{H}|\not D \varphi|^{2} d \Sigma \quad \text { and } \quad\left|\varphi_{H}\right|^{2} d \Sigma_{H}=H|\varphi|^{2} d \Sigma .
$$

Now, Inequality (29) wrt the metric $\langle,\rangle_{H}$ and aaplied to the spinor field $\varphi_{H}$, translates to

$$
0 \leq \int_{\Sigma}\left(\left|\not D_{H} \varphi_{H}\right|^{2}-\frac{n^{2}}{4}\left|\varphi_{H}\right|^{2}\right) d \Sigma_{H}
$$

which is valid for all $\varphi_{H}=H^{-\frac{n-1}{2}} \varphi$, with $\varphi \in \Gamma(\$ \Sigma)$ arbitrary. This is equivalent to the inequality

$$
\lambda_{k}\left(\not D_{H}\right)^{2} \geq \frac{n^{2}}{4}
$$

for all the eigenvalues $\lambda_{k}\left(D_{H}\right)$ of $\not D, k \in \mathbb{Z}$, hence Inequality (3).

If equality holds in (3), then there is a non trivial spinor field $\varphi_{H}=H^{-\frac{n-1}{2}} \varphi \in$ $\Gamma(\$ \$ \Sigma)$ such that $\not_{H} \varphi_{H}=\frac{n}{2} \varphi_{H}$. From (31), this is equivalent to $\not D \varphi=\frac{n}{2} H \varphi$. Then, it is clear that $\varphi$ satisfies the equality in (29). Thus there exist two parallel spinor fields $\psi$ and $\Psi$ on $M$ with $P_{+} \psi=P_{+} \varphi$ and $P_{-} \Psi=P_{-} \varphi$. From (17) and (25), it follows

$$
\frac{n}{2} H P_{-} \psi=\not D P_{+} \psi=\not D P_{+} \varphi=\frac{n}{2} H P_{-} \varphi
$$

Hence, $P_{ \pm} \psi=P_{ \pm} \varphi$, and so $\varphi$ is the restriction to $\Sigma$ of the parallel spinor field $\psi$ on $M$.

As for the connectedness of $\Sigma$ in the equality case, take a non trivial eigenspinor $\varphi_{H} \in \Gamma(\$ \Phi)$ of $\not D_{H}$ associated with the eigenvalue $\frac{n}{2}$. Let $\Sigma_{0}$ be a connected component of $\Sigma$ where $\varphi_{H}$ is non trivial and define a new spinor field $\widetilde{\varphi}_{H}$ on $\Sigma$ in the following way:

$$
\widetilde{\varphi}_{H}= \begin{cases}\varphi_{H} & \text { on } \Sigma_{0} \\ 0 & \text { on } \Sigma-\Sigma_{0} .\end{cases}
$$

It is clear that $\widetilde{\varphi}_{H} \in \Gamma(\$ \Phi)$ and that

$$
\not D_{H} \widetilde{\varphi}_{H}=\frac{n}{2} \widetilde{\varphi}_{H}
$$

that is, $\widetilde{\varphi}_{H}$ is another non trivial eigenspinor of $\not_{H}$ associated with the eigenvalue $\lambda_{1}\left(\not D_{H}\right)=\frac{n}{2}$. Hence, it is the restriction to $\Sigma$ of a non trivial parallel spinor field $\psi$ on $M$ multiplied by the positive function $H^{-\frac{n-1}{2}}$. Since $M$ is connected, the length of $\psi$ is constant and so $\widetilde{\varphi}_{H}$ has no zeros. This implies $\Sigma=\Sigma_{0}$ and then $\Sigma$ is connected.

REMARK 5. Since the Euclidean space $M=\mathbb{R}^{n+1}$ is a spin manifold admitting a

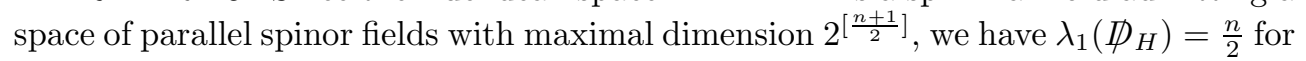


any compact mean convex embedded hypersurface $\Sigma \subset \mathbb{R}^{n+1}$ (which always bounds a compact domain), and the corresponding associated eigenspace is $2^{\left[\frac{n+1}{2}\right]}$-dimensional. Moreover, we deduce that: a compact mean convex hypersurface embedded in $\mathbb{R}^{n+1}$ must be connected. When the hypersurface $\Sigma$ is allowed to have self-intersections, that is, when $\Sigma$ is an orientable hypersurface immersed in $\mathbb{R}^{n+1}$ with nowhere vanishing mean curvature, we proved in $[\mathrm{HM}]$ that $\lambda_{1}\left(\not D_{H}\right) \leq \frac{n}{2}$, and equality implies that the associated eigenspinors correspond to parallel spinors on $\mathbb{R}^{n+1}$.

Proof of Theorem 2. Choose a connected component $\Sigma_{0}$ of $\Sigma$ and define $\varphi \in \Gamma(\$ \Sigma \Sigma)$ to be the restriction to $\Sigma_{0}$ of a non trivial parallel spinor field on $M_{0}$ and to be identically zero on $\Sigma-\Sigma_{0}$. Then, using (17), we have on $\Sigma$

$$
\not D \varphi=\frac{n}{2} H_{0} \varphi
$$

Since $\varphi$ has a non trivial constant length on $\Sigma_{0}$, it is sufficient to apply Inequality (29) to $\varphi$ in order to get Inequality (1) on the component $\Sigma_{0}$. Thus, the same inequality must hold on the whole of the boundary $\Sigma$. Suppose now that equality holds. By Proposition 9 , there exist two parallel spinor fields $\psi, \Psi \in \Gamma(\mathbb{S} M)$ such that $P_{+} \psi=$ $P_{+} \varphi$ and $P_{-} \Psi=P_{-} \varphi$. Then, using (17), (25) and the equality above, we have

$$
H_{0} P_{+} \varphi=\frac{2}{n} \not D P_{-} \varphi=\frac{2}{n} \not D P_{-} \Psi=H P_{+} \Psi \text {. }
$$

Similarly, we obtain

$$
H_{0} P_{-} \varphi=\frac{2}{n} \not D P_{+} \varphi=\frac{2}{n} \not D P_{+} \psi=H P_{-} \psi .
$$

Applying the operator $\not D$ to the first and last terms of (33), we get

$$
\chi_{(}\left(\not \nabla H_{0}\right) P_{+} \varphi+\frac{n}{2} H_{0}^{2} P_{-} \varphi=\not /(\not \nabla H) P_{+} \Psi+\frac{n}{2} H^{2} P_{-} \Psi,
$$

and using again the equalities above, we have finally

$$
\not\left(\not \nabla H_{0}\right) P_{+} \varphi+\frac{n}{2} H_{0}^{2} P_{-} \varphi=\frac{H_{0}}{H} \not(\not \nabla H) P_{+} \varphi+\frac{n}{2} H^{2} P_{-} \varphi .
$$

The same argument applied to (34), yields

$$
\not\left(\not \nabla H_{0}\right) P_{-} \varphi+\frac{n}{2} H_{0}^{2} P_{+} \varphi=\frac{H_{0}}{H} \not(\not \nabla H) P_{-} \varphi+\frac{n}{2} H^{2} P_{+} \varphi .
$$

The sum of the last two formulae, implies

$$
\not /\left(\not H_{0}\right) \varphi+\frac{n}{2} H_{0}^{2} \varphi=\frac{H_{0}}{H} \not(\not \nabla H) \varphi+\frac{n}{2} H^{2} \varphi
$$

Since the spinor fields $\not(\not \nabla H) \varphi$ and $\not\left(\not \nabla H_{0}\right) \varphi$ are both orthogonal to $\varphi$, and the spinor $\varphi$ has non trivial constant length on $\Sigma_{0}$, we finally obtain

$$
\begin{cases}H_{0}^{2} & =H^{2} \\ \not \nabla H_{0} & =\frac{H_{0}}{H} \not \nabla H\end{cases}
$$


on that component $\Sigma_{0}$. From this we conclude that $H_{0}$ has no zeros and so we may assume that

$$
H_{0}=H \text {. }
$$

Coming back now to (33) and (34), we deduce that

$$
\left\{\begin{array}{l}
P_{+} \varphi=P_{+} \Psi \\
P_{-} \varphi=P_{-} \psi
\end{array}\right.
$$

Thus the two parallel spinor fields $\psi, \Psi \in \Gamma(\mathbb{S} M)$ satisfy

$$
\psi_{\mid \Sigma_{0}}=\Psi_{\mid \Sigma_{0}}=\varphi_{\mid \Sigma_{0}} .
$$

By definition, on $\Sigma-\Sigma_{0}$ we have $\varphi_{\mid \Sigma-\Sigma_{0}}=0$. Thus, on $\Sigma-\Sigma_{0}$, one has

$$
P_{+} \psi=P_{+} \varphi=0, \quad P_{-} \Psi=P_{-} \varphi=0 .
$$

Applying the induced Dirac operator $\not D$ to these equalities and taking again into account (17) and (25), we have

$$
0=\not D P_{+} \psi=\frac{n}{2} H P_{-} \psi, \quad 0=\not D P_{-} \Psi=\frac{n}{2} H P_{+} \Psi
$$

We conclude that both parallel spinor fields $\psi$ and $\Psi$ on $M$ vanish on the complement of $\Sigma_{0}$. Since $M$ is connected, $\psi$ and $\Psi$ must have constant length on the whole of $M$. However, this length vanishes on $\Sigma-\Sigma_{0}$ and is a non zero constant on $\Sigma_{0}$. This proves that the boundary hypersurface $\Sigma$ is connected.

As another conclusion, we have that, if equality holds in Inequality (1), the mean curvatures $H$ and $H_{0}$ coincide and that each restriction $\varphi \in \Gamma(\mathbb{\$} \Sigma)$ to $\Sigma$ of a parallel spinor field on $M_{0}$ is the restriction to the boundary hypersurface $\Sigma$ of a parallel spinor field $\psi$ defined on the whole of $M$.

Now we can apply to such a $\varphi \in \Gamma(\$ \Sigma)$ the spinorial Gauß formula (16) for the embedding of $\Sigma$ in $M$ as its boundary and so get

$$
\nabla_{X} \varphi=\nabla_{X}^{M} \psi-\frac{1}{2} \not(A X) \varphi=-\frac{1}{2} \not(A X) \varphi
$$

where $X \in \Gamma(T \Sigma)$. Using again (16) for the immersion of $\Sigma$ into the manifold $M_{0}$, we have

$$
\nabla_{X} \varphi=\nabla_{X}^{M_{0}} \psi_{0}-\frac{1}{2} \not\left(A_{0} X\right) \varphi=-\frac{1}{2} \not\left(A_{0} X\right) \varphi
$$

where $A_{0}$ is the shape operator of this immersion. Since the spinor field $\varphi$ has constant length, we conclude that the two shape operators $A$ and $A_{0}$, corresponding to the embedding of $\Sigma$ in $M$ and to the immersion of $\Sigma$ in $M_{0}$, coincide.

The converse is clear. If the two shape operators $A$ and $A_{0}$ coincide, then the corresponding traces $n H$ and $n H_{0}$ taken with respect to the common induced metric should be equal. From here equality in (1) is straightforward.

Since Euclidean spaces admit non-trivial parallel spinor fields, it is clear that Inequality (1) proved in Theorem 2 is valid when the mean convex boundary hypersurface $\Sigma$ of the $(n+1)$-dimensional Riemannian spin manifold $M$ with non-negative scalar curvature can be immersed, in an isometric and isospin way, in the Euclidean 
space $\mathbb{R}^{n+1}$. In fact, in this particular situation, we may considerably improve the equality case. This is the aim of Theorem 3 .

Proof of Theorem 3. It suffices to study the equality case. From Theorem 2, it is clear that the boundary hypersurface $\Sigma$ has to be connected. On the other hand, since the Euclidean space $\mathbb{R}^{n+1}$ admits a maximal number $2^{\left[\frac{n+1}{2}\right]}$ of linearly independent parallel spinor fields, we can repeat the initial argument in the proof of Theorem 2 for each one of the restrictions to $\Sigma$ of these spinor fields. In this way, we obtain this same number $2^{\left[\frac{n+1}{2}\right]}$ of independent parallel spinor fields defined on the bulk manifold $M$. But, according to [Wa], this maximal number is only attained only by flat manifolds. Thus, $M$ is a flat manifold and so we may see it as an open set of a Euclidean quotient, that is, $M \subset \mathbb{R}^{n+1} / \Gamma$, where $\Gamma$ is a group of Euclidean motions acting properly and discontinuously on $\mathbb{R}^{n+1}$. Let $\widetilde{M} \subset \mathbb{R}^{n+1}$ be any connected component of the lifting of $M$ to the Euclidean space. Then any connected component of the boundary $\widetilde{\Sigma}$ of $\widetilde{M}$ is a connected hypersurface embedded in $\mathbb{R}^{n+1}$ covering the original hypersurface $\Sigma$. Denote by $\pi: \widetilde{\Sigma} \rightarrow \Sigma$ the corresponding projection. Then the composition $F \circ \pi: \widetilde{\Sigma} \rightarrow \mathbb{R}^{n+1}$ is an immersion whose first and second fundamental forms coincide with those of the embedding $\iota: \widetilde{\Sigma} \subset \mathbb{R}^{n+1}$ as the boundary of the domain $\widetilde{M}$. Then we may apply the fundamental theorem of the local theory of surfaces (see, for example, [MR, Theorem 7.7 , p. 209]) to deduce that there exists a rigid motion $\widetilde{F}$ of the Euclidean space $\mathbb{R}^{n+1}$ such that $F \circ \pi=\widetilde{F} \circ \iota$. Hence the covering map $\pi$ is injective. This means that $\widetilde{\Sigma}=\Sigma, \pi=\operatorname{Id}_{\mid \Sigma}$ and $F=\widetilde{F} \circ \iota$.

As we have seen, the proof of Inequality (1) makes no use of the PMT. However, even though it is weaker than Inequality (2), it still implies the PMT, as we will see in the following:

Corollary 10. For any compact connected Riemannian 3-dimensional manifold $M$ with non-negative scalar curvature and mean convex boundary surface $\Sigma$, assume that

$$
\int_{\Sigma} H d \Sigma \leq \int_{\Sigma} \frac{H_{0}^{2}}{H} d \Sigma
$$

where $H$ is the mean curvature of $\Sigma$ as the boundary of $M$ and $H_{0}$ stands for the mean curvature of any immersion $F$ of $\Sigma$ into $\mathbb{R}^{3}$. Let $P$ be a complete non compact 3-dimensional Riemannian asymptotically flat manifold with finitely many ends, with non-negative integrable scalar curvature. Then the ADM mass of each end of $P$ is non-negative.

Proof. Without loss of generality, we may assume that $P$ has only one end, denoted by $E$. Then, we may assume that $E$ is the exterior of a Euclidean ball of $\mathbb{R}^{3}$ and, from the work of Schoen and Yau (see the proof of [ST1, Theorem 5.1] and references therein), the assumption of asymptotically flatness may be translated into the fact that the Riemannian metric $\langle,\rangle_{P}$ on $E$ differs from the Euclidean metric $\langle$,$\rangle in this way:$

$$
\langle,\rangle_{P}(x)=\left(1+\frac{m}{|x|}\right)\langle,\rangle+h_{x}, \quad \forall x \in E \subset \mathbb{R}^{3},
$$

where $h_{x}$ is a symmetric bilinear form satisfying

$$
\left|\frac{\partial^{k} h}{\partial x_{i}}(x)\right|=O\left(\frac{1}{|x|^{2+k}}\right), \quad i=1, \ldots, 3, \quad k=0, \ldots, 4,
$$


for all $x \in E$, and where the constant $m \in \mathbb{R}$ is just the ADM mass of the end $E$. Thus, we aim to prove that $m \geq 0$.

For each $r>0$, large enough, take the Euclidean sphere $\mathbb{S}_{r}^{2} \subset E$ of radius $r$ centered at the origin. Consider now the compact 3 -dimensional manifold $M$ obtained by taking off from $P$ the exterior domain determined by $\mathbb{S}_{r}^{2}$. Then $M$ is a compact connected Riemannian manifold with non-negative scalar curvature and whose boundary is just the surface $\mathbb{S}_{r}^{2}$. Recall also that $M$, like all 3-dimensional manifolds, is spin. In order to apply to $M$ the integral inequality that we assumed, we need to compute the inward mean curvature $H$ of $\mathbb{S}_{r}^{2}$ with respect to the metric $\langle,\rangle_{P}$. In fact, this more or less straightforward computation can be found in [ST1]. More precisely, they proved that

$$
H=\frac{1}{r}-\frac{2 m}{r^{2}}+O\left(\frac{1}{r^{3}}\right), \quad K=\frac{1}{r^{2}}-\frac{2 m}{r^{3}}+O\left(\frac{1}{r^{4}}\right),
$$

where $K$ is the Gauß curvature of $\mathbb{S}_{r}^{2}$ with the metric induced from $\langle,\rangle_{P}$. This proves that, for $r$ large enough, not only the boundary surface $\mathbb{S}_{r}^{2}$ is mean convex, that is $H>0$, but also that it is strictly convex, that is, $K>0$. This last property guarantees, by applying the Weyl Embedding Theorem (see [We]) that the sphere $\mathbb{S}_{r}^{2}$ can be embedded in $\mathbb{R}^{3}$ in such a way that the metrics induced on $\mathbb{S}_{r}^{2}$ from $\mathbb{R}^{3}$ and the one induced from $M$ coincide. Moreover, this embedding is unique up to Euclidean congruences. This means that the mean curvature $H_{0}$ of this embedding is completely determined by this metric. In fact, by using the estimates for $H_{0}$ in terms of $K$ obtained by Weyl, Shi and Tam got the following estimate at infinity (see [ST1] having in mind that their mean curvatures are not normalized)

$$
H_{0}=\frac{1}{r}-\frac{m}{r^{2}}+O\left(\frac{1}{r^{3}}\right) .
$$

From the asymptotic expressions for $H$ and $H_{0}$ above, it is immediate to conclude that

$$
\frac{H_{0}^{2}}{H}=\frac{1}{r}+O\left(\frac{1}{r^{3}}\right)
$$

Finally, in order to compute the integrals on $\mathbb{S}_{r}^{2}$ of the two functions $H$ and $H_{0}^{2} / H$, we need some information about the Riemannian measure $d \mathbb{S}_{r}^{2}$. It is clear that the map $F: \mathbb{S}^{2} \rightarrow \mathbb{S}_{r}^{2}$ given by $F(y)=r y$ for $y \in \mathbb{S}^{2}$ is a diffeomorphism and can be used as a parametrization of $\mathbb{S}_{r}^{2}$. We can see in $[\mathrm{ST} 1,(5.5)]$ that

$$
F_{r}^{*}\left(d \mathbb{S}_{r}^{2}\right)=\left(r^{2}+2 m r+O(1)\right) d \mathbb{S}^{2},
$$

where $d \mathbb{S}^{2}$ is the Riemannian measure of the Euclidean unit sphere. Now, we may write asymptotic expressions for the integrals on the sphere $\mathbb{S}_{r}^{2}$ of the three functions $H, H_{0}$ and $H_{0}^{2} / H$. In fact, we have

$$
\begin{aligned}
\int_{\mathbb{S}_{r}^{2}} H d \mathbb{S}_{r}^{2} & =4 \pi\left(r+O\left(\frac{1}{r}\right)\right) \\
\int_{\mathbb{S}_{r}^{2}} H_{0} d \mathbb{S}_{r}^{2} & =4 \pi\left(r+m+O\left(\frac{1}{r}\right)\right) \\
\int_{\mathbb{S}_{r}^{2}} \frac{H_{0}^{2}}{H} d \mathbb{S}_{r}^{2} & =4 \pi\left(r+2 m+O\left(\frac{1}{r}\right)\right),
\end{aligned}
$$


for all $r>0$ large enough. Now, one can see that Inequality (1), assumed to be true as a hypothesis, with $\Sigma=\mathbb{S}_{r}^{2}, r>0, r \rightarrow \infty$, implies that $m \geq 0$.

REMARK 6. Note that, in Corollary 10, we can substitute Inequality (1) either by the Shi and Tam Inequality (2), which is valid only under convexity assumptions for $\Sigma$, or by Inequality (5), which is valid only when the integral on $\Sigma$ of the mean curvature $H_{0}$ is positive. The PMT can be deduced from any of these three inequalities for the boundary of a compact connected 3-dimensional manifold with non-negative scalar curvature. The crucial difference is that the Shi and Tam inequality is proved using the PMT as one of its key ingredients, and also that the realm of potential applications of the other two is wider.

From Theorem 3, it is not difficult to deduce as well a congruence result for Euclidean immersions of mean convex boundaries of compact connected Riemannian spin manifolds with non-negative scalar curvature. This result (see Corollary 11) generalizes that of Ros $([\mathrm{R}])$ where the bulk manifold is supposed to have non-negative Ricci curvature. In $[R]$, this congruence result is presented as a generalization of an old rigidity theorem proved by Schur for plane Euclidean curves. This same generalization is obtained in $[\mathrm{HW}]$, where it is presented as a solution to a conjecture by Schroeder and Strake, and also in [EMW, Lemma 5].

Corollary 11. Let $M$ be an $(n+1)$-dimensional compact connected Riemannian spin manifold with non-negative scalar curvature and mean convex boundary hypersurface $\Sigma$. Suppose that $\Sigma$ admits an isometric and isospin immersion $F$ into the Euclidean space $\mathbb{R}^{n+1}$ and that the mean curvature $H$ of $\Sigma$ as the boundary of $M$ and the mean curvature $H_{0}$ of the immersion $F$ of $\Sigma$ in $\mathbb{R}^{n+1}$ satisfy the pointwise inequality $\left|H_{0}\right| \leq H$. Then, $M$ is a Euclidean domain with connected boundary. Moreover, the embedding of $\Sigma$ in $M$ as its boundary and the immersion of $\Sigma$ in $\mathbb{R}^{n+1}$ are congruent.

Proof. Since we assume that the inequality $\left|H_{0}\right| \leq H$ holds, we have that

$$
\int_{\Sigma} \frac{H_{0}^{2}}{H} d \Sigma \leq \int_{\Sigma} H d \Sigma
$$

and so equality is attained in Inequality (1).This finishes the proof.

\section{REFERENCES}

[APS] M. F. Atiyah, V. K. Patodi, And I. M. Singer, Spectral asymmetry and Riemannian geometry, I, Math. Proc. Cambridge Phil. Soc., 77 (1975), pp. 43-69.

[Bä1] C. Bär, On nodal sets for Dirac and Laplace operators, Commun. Math. Phys., 188 (1997), pp. 709-721.

[Bä2] C. B̈̈r, Extrinsic bounds of the Dirac operator, Ann. Glob. Anal. Geom., 16 (1998), pp. $573-596$.

[BäBa] C. Bär AND W. Ballmann, Guide to boundary value problems for Dirac-type operators, arXiv:1307.3021 [math.DG].

[BFGK] H. BAum, T. Friedrich, R. Grünewald, and I. Kath, Twistor and Killing Spinors on Riemannian Manifolds, Seminarbericht 108, Humboldt-Universität zu Berlin, 1990.

[BC] R. BARTNik And P. Chruściel, Boundary value problems for Dirac-type equations, J. reine angew. Math., 579 (2005), pp. 13-73.

[BW] B. Booss-Bavnbek And K. P. Wojciechowski, Elliptic Boundary Problems for the Dirac Operator, Birkhäuser, Basel, 1993. 
[BHMmM] J. P. Bourguignon, O. Hijazi, J.-L. Milhorat, A. Moroianu, and S. Moroianu, A Spinorial Approach to Riemannian and Conformal Geometry, to appear in Monographs in Mathematics, European Mathematical Society (2014).

[BY] J. BRown AND J. YORK, Quasilocal energy and conserved charges derived from the gravitational action, Phys. Rev. D, 3 47(4) (1993), pp. 1407-1419.

[Bu] J. Burěs, Dirac operators on hypersurfaces, Comment. Math. Univ. Carolin., $34: 2$ (1993), pp. 313-322.

[CJjtw] A. Chodos, R. L. Jaffe, K. Johnson, C. B. Thorn, and V. F. Weisskopf, New extended model of hadrons, Phys. Rev. D, 9 (1974), pp. 3471-3495.

[CJJT] A. Chodos, R. L. Jaffe, K. Johnson, and C. B. Thorn, Baryon structure in the bag theory, Phys. Rev. D, 10 (1974), pp. 2599-2604.

[EMW] M. Eichmair, P. Miao, And X. Wang, Extension of a theorem of Shi and Tam, Calc. Var., 43 (2012), pp. 45-56.

[GLP] P. B. Gilkey, J. V. LeAhy, AND J. PARK, Spectral geometry, Riemannian submersions and the Gromov-Lawson conjecture, Studies in Advanced Mathematics, Chapman \& Hall/Crc, Boca Raton, 1999.

[HW] F. HANG AND X. WANG, A note on a conjecture of Schroeder and Strake, Pacific Math. J., 232 (2007), pp. 283-287.

[He] M. Herzlich, The positive mass theorem for black holes revisited, J. Geom. Phys., 26 (1998), pp. 97-111.

[Hij] O. HiJAzI, A conformal lower bound for the smallest eigenvalue of the Dirac operator and Killing spinors, Commun. Math. Phys., 104 (1986), pp. 151-162.

[HM] O. Hijazi And S. Montiel, A spinorial characterization of hyperspheres, Calc. Var., 48 (2013), pp. 527-544.

[HMR] O. Hijazi, S. Montiel, And A. Roldán, Eigenvalue boundary problems for the Dirac operator, Comm. Math. Phys., 231 (2002), pp. 375-390.

[HMZ1] O. HiJazi, S. Montiel, AND X. Zhang, Dirac operator on embedded hypersurfaces, Math. Res. Lett, 8 (2001), pp. 195-208.

[HMZ2] O. HiJazi, S. Montiel, AND X. Zhang, Eigenvalues of the Dirac operator on manifolds with boundary, Commun. Math. Phys., 221 (2001), pp. 255-265.

[HMZ3] O. Hijazi, S. Montiel, And X. Zhang, Conformal lower bounds for the Dirac operator of embedded hypersurfaces, Asian J. Math., 6 (2002), pp. 23-36.

[Hit] N. Hitchin, Harmonic spinors, Adv. in Math., 14 (1974), pp. 1-55.

[Hö] L. Hörmander, The Analysis of Linear Partial Differential Operators III, Springer, Berlin, 1985.

H. B. Lawson and M. L. Michelsohn, Spin Geometry, Princeton Math. Series, vol. 38, Princeton University Press, 1989.

C.-C. Liu And S.-T. Yau, Positivity of quasilocal mass, Phys. Rev. Lett., 90 (2003), pp. 231102-231106.

C.-C. LiU And S.-T. Yau, Positivity of quasi-local mass II, J. Amer. Math. Soc., 19:1 (2006), pp. 181-204.

S. Montiel And A. Ros, Curves and Surfaces, $2^{\text {nd }}$ edition, Graduate Studies in Mathematics, 69 (2009), American Mathematical Society, Rhode Island.

A. Ros, Compact hypersurfaces with constant scalar curvature and a congruence theorem, J. Diff. Geom., 27 (1988), pp. 215-220.

Y. SHI AND L.-F. TAM, Positive mass theorem and the boundary behaviors of compact manifolds with nonnegative scalar curvature, J. Diff. Geom., 62 (2002), pp. 79-125.

Y. SHI AND L.-F. TAM, Rigidity of compact manifolds and positivity of quasi-local mass, Classical Quantum Gravity, 24 (2007), pp. 2357-2366.

Y. SHi AND L.-F. TAM, Quasi-local mass and the existence of horizons, Comm. Math. Phys., 274 (2007), pp. 277-295.

R. Schoen And S.-T. YAu, On the proof of the positive mass theorem conjecture in general relativity, Commun. Math. Phys., 65 (1979), pp. 45-76.

R. SEeley, Singular integrals and boundary problems, Amer. J. Math., 88 (1966), pp. $781-809$.

A. Trautman, The Dirac operator on hypersurfaces, Acta Phys. Pol., B26 (1995), pp. 1283-1310.

pp. 59-68.
[We] H. WEYL, Über die Bestimmung einer geschlossenen konvexen Fläche durch ihr
Linienelement, Vierteljahrsschrift der naturforschenden Gesellschaft, 61 (1916), 
pp. $40-72$

M.-T. WANG AND S.-T. YAU, A generalization of Liu-Yau's quasi-local mass, Comm. Anal. Geom., 15 (2007), pp. 249-282.

[Wi] E. Witten, A new proof of the positive energy theorem, Commun. Math. Phys., 80 (1981), pp. 381-402. 